\title{
Computer Experiments of an Electron Beam Injection into Space Plasma
}

\author{
H. Matsumoto ${ }^{1}$, N. Komori ${ }^{1}$, M. Ashour-Abdalla ${ }^{2}$, and D. Donatellit ${ }^{3}$ \\ ${ }^{1}$ Radio Atmospheric Science Center, Kyoto University, Uji, Kyoto 611, Japan \\ ${ }^{2}$ Institute of Geophys. and Planetary Physics and Physics Department, UCLA, Los Angeles, \\ California 90024, U.S.A. \\ ${ }^{3}$ Air Force Geophysics Laboratory, Hanscom AFB, Bedford, Massachusetts 01731, U.S.A.
}

(Received October 30, 1987; Revised January 5, 1988)

\begin{abstract}
A computer experiment is carried out to study the dynamic behavior of plasma particles in response to self-consistent electromagnetic and electrostatic fields produced by an artificial injection of a dense electron beam into the ambient plasma. A two-and-one-half dimensional electromagnetic particle code (KEMPO) is used for this purpose. The electron beam is assumed to be injected along the external magnetic field, $B_{0}$, and to have a spatially finite extent in directions both parallel and perpendicular to the beam motion at $t=0$. Most of the beam electrons are decelerated, and this deceleration is accompanied by an elongation and splitting of the beam. However, a fraction of the electrons in the beam is not decelerated but instead is accelerated in both positive and negative directions along $B_{0}$. A substantial heating of beam electrons is also observed. Associated with the acceleration and thermalization of the beam electrons, various kinds of electromagnetic and electrostatic waves are excited through wave-particle interactions. On a time scale of ion motion, ions are attracted toward the center of the moving beam, forming an ion density peak, which, in turn, causes a strong acceleration of electrons. A BGK type quasi-steady state is reached and maintained for a finite time.
\end{abstract}

\section{Introduction}

Active experiments in space using particle beams from space vehicles have recently attracted interest in both plasma and space physics. Applications of these beam experiments in space are classified into two different categories. One is the use of electron beams as remote probes of the electric and magnetic field configuration of the distant magnetosphere (such as ECHO series rocket experiments, see e.g., WINCKLER (1982)) and as the in-situ probe in place of the probe (antenna) measurement of the DC electric field (such as GEOS $E$-field measurement, BAUMJOHANN et al. (1985) and BAUMJOHANN and HAERENDEL (1985)). The other is the use of beams as artificial free energy sources with known properties and thus to collect information on wave-particle interactions with an aim at understanding natural beam phenomena such as particle precipitation in the auroral region and particle acceleration in the geotail region. Space experiments in the second category 
provide an opportunity of studying nonlinear dynamics of particle acceleration and heating as well as the associated wave generation under various controlled conditions. A variety of experimental output has been obtained thus far by these active experiments (CARTWRIGHT and KELLOG, 1974; WINCKLER et al., 1975, 1984, 1985; MATSUMOTO et al., 1975; KAWASHIMA et al., 1981; WINCKLER, 1982; SHAWHAN et al., 1984; OBAYASHI et al., 1985; ARNOLDY et al., 1985). However, the results of these experiments were generally more complicated than were expected, and as a consequence, the theoretical analysis was difficult. The difficulties come not only from the complexity inherent in the nonlinearity and inhomogeneity of wave-particle interactions due to the local injection of particle beams, but also from experimental limitations and restrictions of instrumental arrangement. Most active experiments are not equipped with a free-flier probe which is capable of measuring particles and waves at a distance from the particle gun. Therefore a theoretical analysis using the limited data obtained only at the site of the gun is extremely difficult and sometimes impossible. However, such unexpected and unexplained space observations brought by the beam injection definitely stimulate a new area of theoretical research (e.g., OBAYASHI et al. (1985) and ARNOLDY et al. (1985) for energization process by beam injection).

Computer simulations become valuable complimentary numerical experiments to such space vehicle experiments. They provide detailed information on particle dynamics and associated nonlinear phenomena for a given model and under given initial conditions which are appropriately justified by the particular space vehicle experiments. Particle simulations for beam experiments have so far been made by focussing on electromagnetic wave emission from an injected beam (OMURA and MATSUMOTO, 1984) and on particle acceleration by UHR and LHR waves excited by an injected electron beam (MATSUMOTO and FUKUCHI, 1985). In the latter simulation both electrostatic and electromagnetic waves are treated, as well as particle dynamics. However, it treated only one spatial dimension perpendicular to the magnetic field while neglecting the parallel motion of the beam. In the present paper we will show the results of a two-and-one-half dimensional particle simulation of the beam injection problem. The main emphasis is on the nonlinear evolution of a finite-sized electron beam, and the associated wave excitation and the particle acceleration/thermalization phenomena in directions both parallel and perpendicular to the external magnetic field.

The motivation of the present study came from unexplained experimental results found in the ARAKS rocket experiment, SEPAC Space Shuttle Electron Beam Experiment and Spacelab-1 Electron Beam Experiment. In these experiments many puzzling facts were discovered such as the beam retardation without loss of beam energy, (ARAKS, LAVERGNAT (1982)) creation of energized high-pitch angle electrons (SEPAC, OBAYASHI et al. (1985); WILHELM et al. (1984a, b)) by the electron beam injection and backward scattering of the injected electron beam (Spacelab-1; Banks (private communication)). The relation of the present simulation with these experiments will be discussed in Section 4.

Section 2 describes the model and code used for the present simulation. Results 
of the simulation are given in Section 3 followed by a discussion and conclusion in Section 4. In Section 4 where we compare the simulation results with LYATSKIY's (1981) and MAL'TSEV's $(1982,1983)$ analytic theories on spatially bounded electron beams.

\section{Model and Simulation Code}

In both categories of the beam experiments in space mentioned in the previous section, the dynamical behavior of the beam itself should be well-understood. The use of the beam as a remote probe for the measurement of the electric and magnetic fields has mostly been based on the theoretical assumption of a dilute beam $\left(n_{\mathrm{b}}<n_{0}\right.$ or $\left.n_{\mathrm{b}} \ll n_{0}\right)$ so that the beam instability or beam boundary effects do not change the properties of the background plasma and fields, where $n_{\mathrm{b}}$ and $n_{0}$ are the densities of the beam and background plasma, respectively. In most of the past experiments such as ECHO series rocket experiments (see review by WINCKLER (1982)) carried out in the ionosphere, this assumption was valid and useful for their theoretical analysis. However, in the distant area such as the magnetosphere, plasma sheet and magnetotail where the background plasma density is extremely low, the beam relative density exceeds unity (i.e., $n_{\mathrm{b}}>n_{0}$ ) because the beam density itself cannot be kept low enough as the beam density should be above a certain threshold of the minimum detectable density. Therefore the study of the dynamical self-consistent behavior of an overdense beam may be useful in such a case. In the other category of space beam experiments, the injected beam is used as a source of beam instabilities. One important point which needs attention is that the artificial electron beam injected from the space vehicle has normally a finite size in both radial and axial irections. Therefore the resultant beam-plasma interactions and instabilities are different from those for the infinite size beam as is often assumed in the analytic theories. The difference comes from the boundary effects such as the finite radial size and frontal and rear edge effects. Therefore the computer experiment of a finite sized electron beam may be a good complimentary study of the active beam experiments in space.

In order to model such a spatially finite electron beam, we adopted a two dimensional beam cloud with finite sizes in both parallel and perpendicular directions of the beam drift velocity as depicted in Fig. 1. To model the spatially finite beam along the beam drift direction, one may adopt a semi-infinite model in which the beam is injected from one end of the simulation box and watch the time evolution of the injected beam including the beam front. However, to avoid a numerical disturbance at the injection point at the end of the simulation area, and to attempt to observe the boundary effects at both ends of the finite-sized electron beam, we did not use this model but hired a model of a finite sized electron cloud in a periodic model. Due to the limit of the available memory in the current our super-computer, the length of the beam along the beam drift direction is not long enough. This may enhance the couplings between the frontal and rear edge effects compared with the more realistic longer beam. Regardless of the short size of the finite beam, the present model gives a lot of information on the micro-physics of the interaction of the finite-sized beam with 


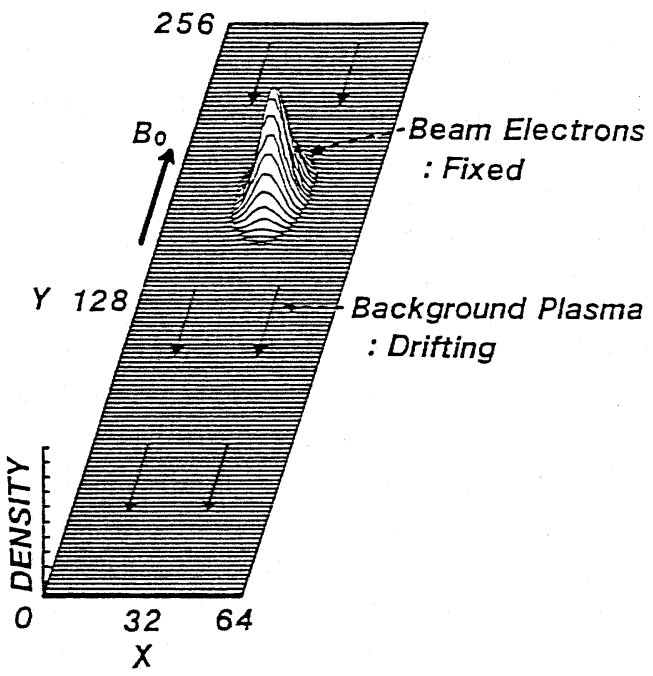

Fig. 1. Simulation model. Spatial coordinates and beam number density at $t=0$ are shown. The external magnetic field is assumed to be parallel to the $y$-axis. The electron beam is spatially isolated and forms an island. The coordinates are in the frame in which the beam is at rest and the background plasma is drifting in the $-y$ direction at the initial beam speed.

a background plasma. The results of another simulation for a longer beam, which aimed to study the propagation effects of the beam through the plasma, will be published elsewhere.

The artificial beam injection from the space vehicle into the surrounding space plasma has two different aspects. One is the current injection and the other is the charge injection. Strong current injection as carried out in the SEPAC experiment on the space shuttle produces a large local excess negative charge in the vicinity of the injection point. The relative beam density of the SEPAC electron beam is approximately four times higher than the back ground density in the vicinity of the gun assuming the electron beam radius is about $10 \mathrm{~cm}$ (OBAYASHI et al., 1985; MATSUMOTO and FUKUCHI, 1985). The excess charge produces various effects through its electrostatic potential until the charge neutrality is achieved. Actually in a very short time after the gun firing, many interesting phenomena such as the production of high energy electrons with very high pitch angles were discovered in the SEPAC. In order to see these transient effects, we adopted a model of the electron cloud carrying an excess charge and placed it in the background plasma as shown in Fig. 1.

In our simulation model we treat the case in which an electron beam is injected along the external magnetic field. We adopt a moving frame with a velocity identical to the beam initial drift velocity $V_{\mathrm{d} 0}$. Therefore the beam electrons in this frame stand 
still in space unless their parallel velocities are modified from their initial values. The background electrons and ions, on the other hand, have an initial speed of $-V_{\mathrm{d} 0}$ along the external magnetic field. The two-dimensional model space is divided into $64 \times 256$ cells with equal grid spacings. The longer $y$-axis is taken along the external magnetic field $B_{0}$.

The simulation code used in this study is a full electromagnetic particle code (KEMPO) which solves the full set of Maxwell's equations and simultaneous equations of motion for many super-particles (see details in MATSUMOTO and OMURA (1985)). The total number of super-particles traced in the simulation is 163,840 . This would correspond to four background electrons, four background ions and two beam electrons per cell if they were distributed uniformly in space. The electron to ion mass ratio is assumed to be 10 , which is much less than the real mass ration but still enables us to distinguish between ion and electron dynamics due to their significantly different time scales. The initial values of the plasma frequencies sued in the present simulation are: background electrons, 2.0; ions, 0.648; and beam, 0.44 . Note that this value of the beam plasma frequency is calculated with the assumption that the beam electrons are distributed uniformly. The actual initial shape of the beam in space, however, is Gaussian in the $x-y$ plane as illustrated in Fig. 1. Therefore the actual beam density at its peak is four times higher than the background plasma density. It is noted that total charge neutrality in the system is conserved, but it is violated locally in the vicinity of the beam at $t=0$. This means that the electron beam is assumed to have been injected into the plasma at $t=0$ and that the local excess charge is balanced by a self-consistent electrostatic electric field. The magnitude of the external magnetic field is taken to be unity, which gives an electron cyclotron frequency of 1.0 for the super-electrons with a charge-to-mass ratio of -1 . The light speed is taken to be 50.0, while the drift velocity of the background plasma with respect to the beam is -5.0 along the $y$-axis. The thermal speed of the background electrons is 1.0 , and that of the beam electrons is 0.707 ; and both are assumed to be isotropic. The Debye length is thus 0.5 . The thermal velocity of the background ions is 0.224 and isotropic. The time step is chosen to be 0.01 while the grid spacing is unity, satisfying the CFL condition for numerical stability.

\section{Simulation Results}

As the system carries the free energy of the drifting beam, we expect the excitation of various kinds of waves with a modification of the particle velocity due to the wave fields. Figure 2 shows the dispersion analysis of the simulation data on the wave fields, which are excited as a result of the interaction of the beam with the plasma. Four kinds of natural wave modes are examined: the electron plasma wave, the Upper Hybrid Resonance (UHR) wave, the electromagnetic $X$ mode wave and the whistler mode wave. The maximum intensity of the spectrum is indicated in the upper right corner of each $\omega-k$ diagram by "MAX". The "ANGLE" shown in the upper left corner indicates the angle of propagation relative to $B_{0}$. As seen in these frames, all wave modes excited by the beam have relatively small $k$ values. Of these waves, the 

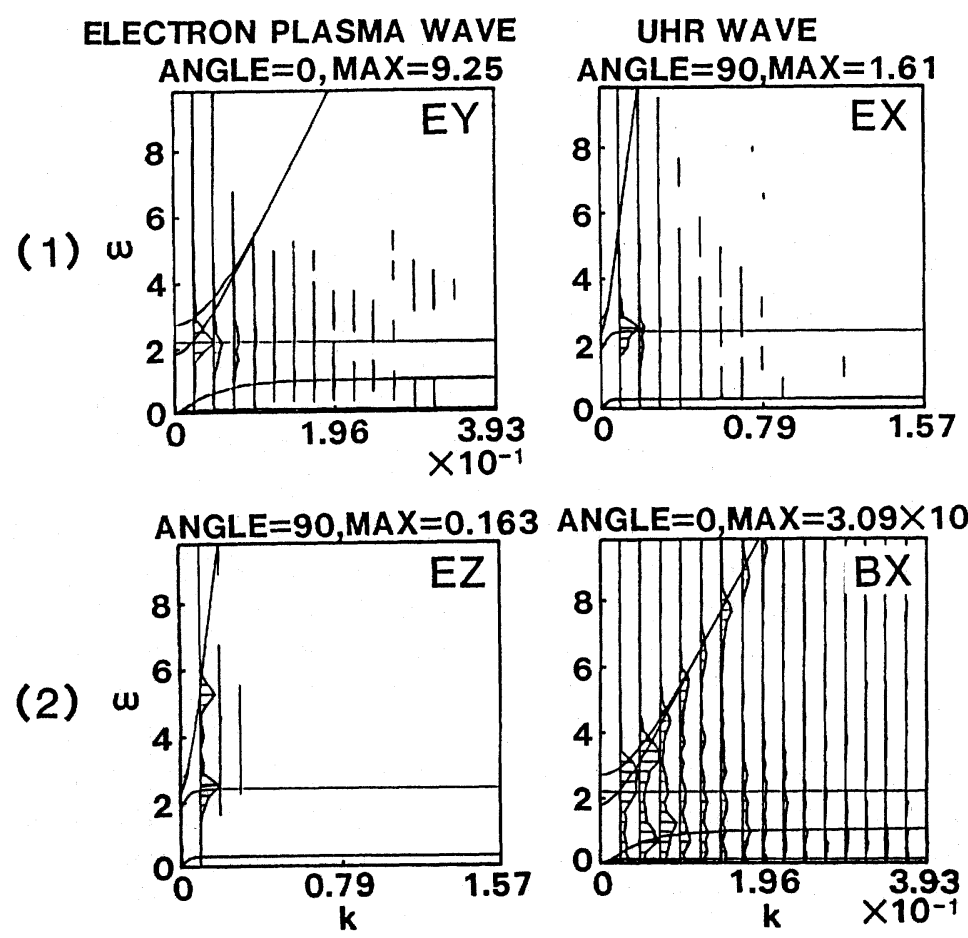

ELECTROMAGNETIC $\mathrm{X}$-mode WAVE

$A N G L E=0, M A X=3.09 \times 10^{-4}$

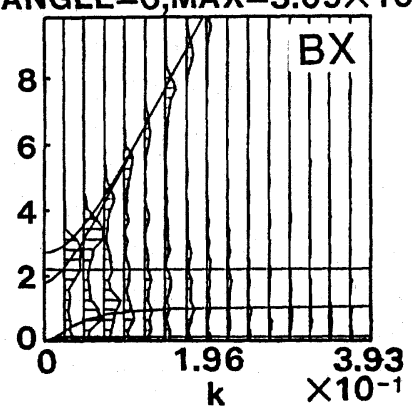

Fig. 2. The $\omega-k$ diagrams are given for four different natural wave modes from the dispersion analysis of the electric and magnetic fields. The solid lines are the dispersion curves calculated using linear theory.

most intense are the electron plasma waves propagating along the external magnetic field. The second most intense are the UHR waves followed by the electromagnetic $X$-mode waves. The weakest waves observed are whistler mode waves.

Figure 3 illustrates the spatial structures and time evolution of the electric fields of the two most intense wave modes ( $E_{y}$ and $E_{x}$, for the electron plasma waves and the UHR waves, respectively). $E_{x}$ is plotted against the $x$-axis on the fixed line of $y=180$, while $E_{y}$ is plotted against the $y$-axis on the fixed line $x=32$. As indicated in the center panel of the figure, both lines pass through the center of the initial beam location. The variation in the spatial structure of $E_{x}$ and $E_{y}$ with time is shown in both panel by shifting along the vertical time axis. These electric fields, $E_{y}$ and $E_{x}$ are basically created by the charge carried by the electron beam. The initial structure of both $E_{y}$ and $E_{x}$ is determined by the Poisson equation, which satisfies the given condition of the initial beam charge centered at $(x, y)=(32,180)$. These electrostatic electric fields show the oscillation modes resulting from the excitation of electrostatic waves in directions both parallel and perpendicular to the external magnetic field. The dispersion analysis 


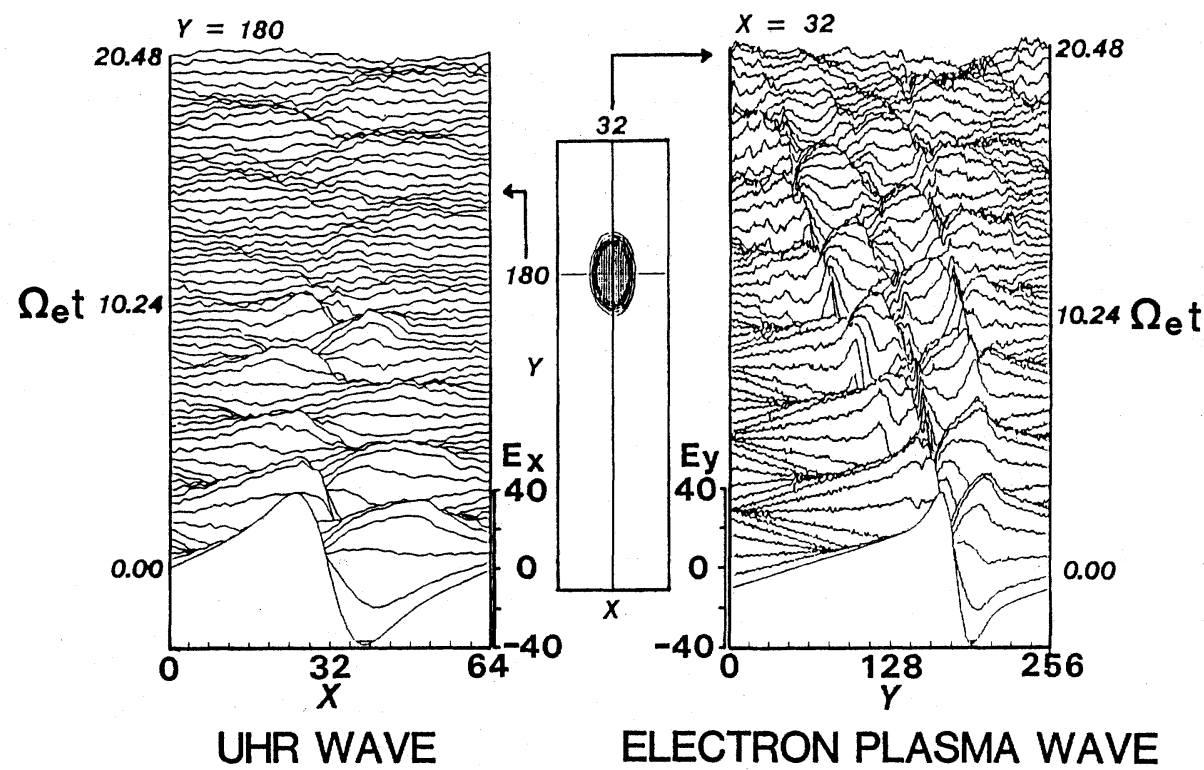

Fig. 3. Time evolution of $E_{x}$ along $x$ for fixed $y$ and $E_{y}$ along $y$ for fixed $x$ for UHR and electron plasma waves, respectively.

illustrated in Fig. 2 shows that waves with $E_{y}$ are electron plasma waves, while those with $E_{x}$ are UHR waves. For the UHR waves, the dominant Fourier mode is a fundamental with a wavelength equal to the system size along the $x$-axis. The UHR waves at $y=180$ eventually attenuate as time elapses. This is due to a shift of the source of the wave along $B_{0}$, away from $y=180$. The nature of the UHR oscillations is essentially the same as that of the UHR waves observed in a previous one-dimensional simulation (MATSUMOTO and FUKUCHI, 1985). The electron plasma waves, on the other hand, show an interesting feature. A significant electric field at $t=0$ is concentrated in the vicinity of the beam, though a small tail-like field component is associated outside the beam region. This is due to the periodic boundary condition imposed on the present model. However, in contrast to the electric field of the UHR waves, $E_{x}$ in the left panel, the electric field $E_{y}$, in the right panel, does not show simple oscillations but instead shows a complicated structure and time evolution. The most intense electric field moves to the left as time elapses. Concurrently, the amplitude of the electric field decreases as the field structure changes into multiple peaks as seen in the figure. These two panels for $E_{x}$ and $E_{y}$ suggest that the beam moves downward along the $y$-axis with deformation of beam shape.

The profiles of the beam density along the $y$-axis at two different $x$ locations, $x=32$ (center of the beam) and $x=20$ (off-center), are plotted as a function of time in Fig. 4. At an early phase, the density profile $x=32$ along the $y$-axis shows a repetitive 


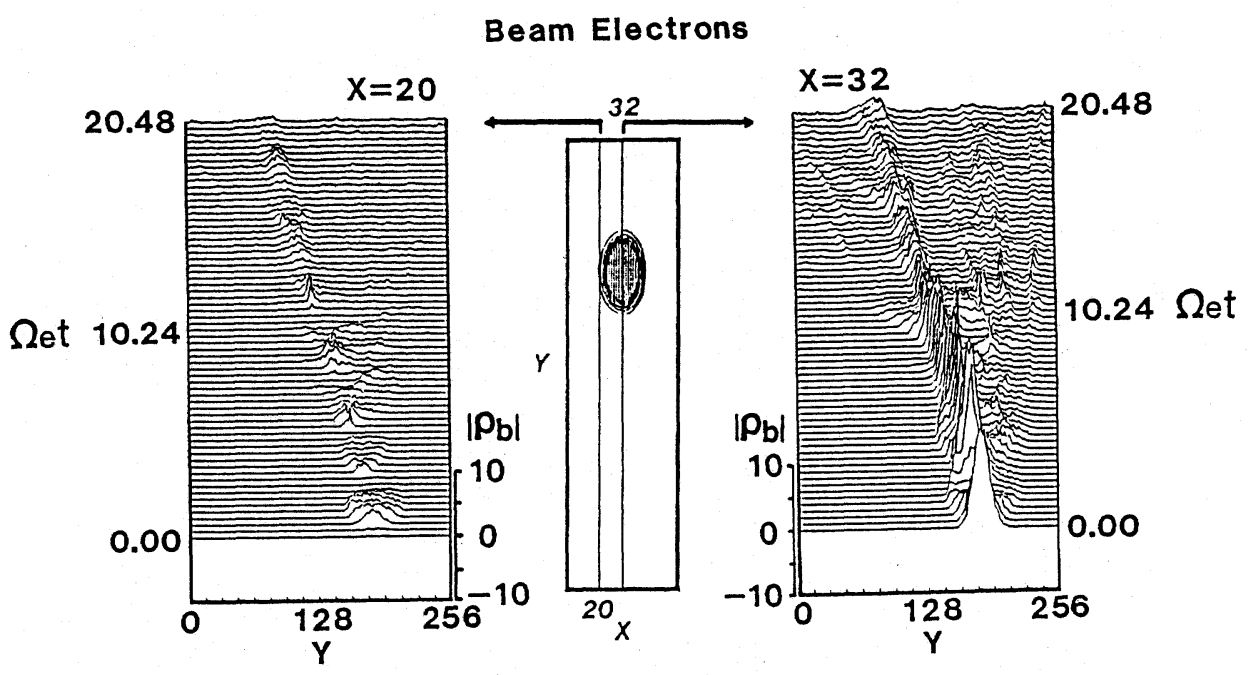

Fig. 4. Time evolution of the beam density profile along the $y$-axis for two fixed $x$ coordinates.

change between a sharp monopeak structure and a less-dense (flattened) structure. Where the profile is flattened, at $x=32$, the profile at $x=20$ shows an enhancement of the beam density in the vicinity of the beam center along the $y$-axis. This means that the beam expands not only in the $y$ direction but in the $x$ direction as well, due to $E_{x}$. This reflects the dynamic reaction of the beam electrons to the electrostatic electric fields $E_{y}$ and $E_{x}$, showing motion consistent with the excitation of the electron plasma waves and the UHR waves. Another point which we need to note is that the monopeak at $X=32$ (corresponding approximately to the center of the beam) moves toward the left along the $y$-axis showing a deceleration of the electron beam due to the interaction with the self-consistent electric field. The locus of the beam center of gravity (defined as the average location of all beam electrons) along the $y$-axis as a function of time is shown by a solid line in Fig. 5(a). The corresponding locus of the center of the main portion of the beam (i.e., the most dense region of the beam) is plotted by a chain line. The dashed line in the same frame indicates the locus of a point moving with the speed of the background plasma, i.e., $v_{y}=-5.0$. The locus of the center of gravity finally reaches an intermediate velocity between the original speed $\left(v_{y}=0\right)$ and the background plasma velocity $\left(v_{y}=-5\right)$. However, the main core is decelerated and finally stops in the background plasma. As seen in Fig. 5(a), the locus does not show a simple monotonic shift with decreasing $y$ but is associated with an oscillating change. This comes from the oscillating nature of the velocity modulation in $\left\langle v_{y}\right\rangle$ which is shown in Fig. 5(b). The oscillation period is between the plasma period determined by the background plasma density $\left(\Omega_{\mathrm{e}} t=3.14\right)$ and that determined by the sum of the background and smeared-out beam electron densities $\left(\Omega_{\mathrm{e}} t=3.07\right)$. This 


\section{Beam Electrons}
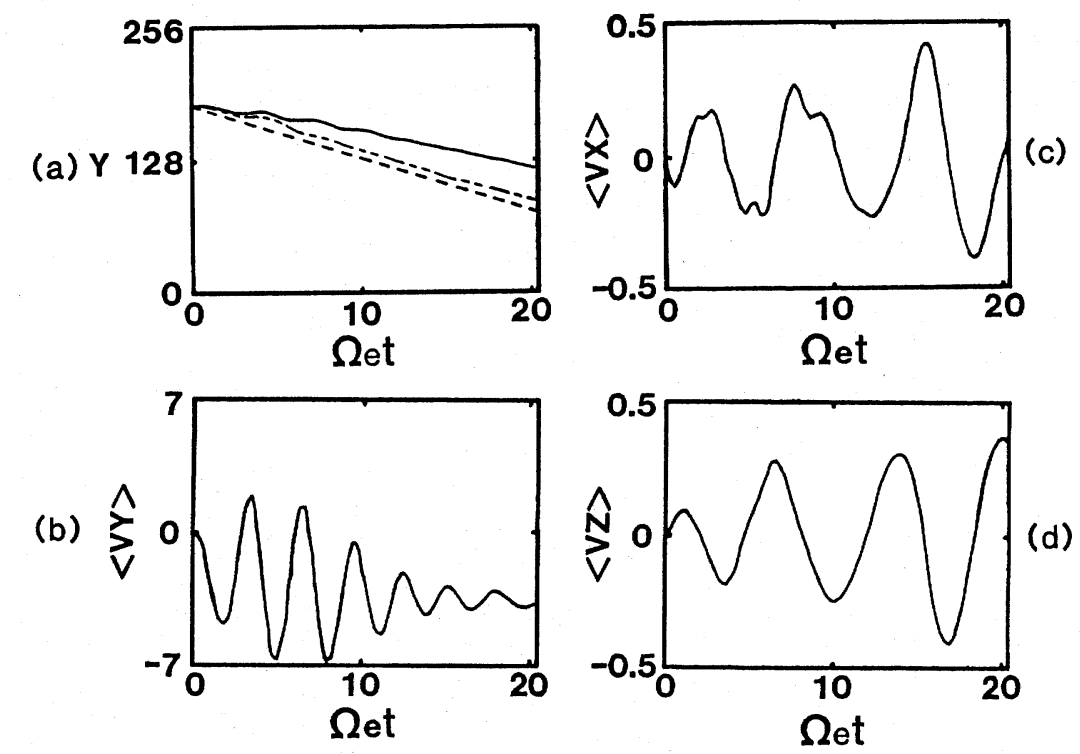

Fig. 5. The average orbit of the beam electrons $Y(=\langle y\rangle)$ and average velocities $\left\langle v_{y}\right\rangle,\left\langle v_{x}\right\rangle$ and $\left\langle v_{z}\right\rangle$ of the beam electrons, are shown as a function of time.

means that the oscillating nature of the beam velocity is a reflection of the plasma oscillation. In Figs. 5(c) and (d), the time behavior of the average velocity of the beam electrons in the perpendicular plane is shown. The oscillation seen in $\left\langle v_{x}\right\rangle$ is not of a pure sinusoidal nature but seem to be a superposition of multi-frequency components as inferred from the $\omega-k$ diagram for $E_{x}$ and $B_{x}$ in Fig. 2. The lowest frequency component is approximately equal to the electron cyclotron frequency. The lower frequency component is also seen in $\left\langle v_{z}\right\rangle$ with a 90 degree phase lag. This is an indication that the lower frequency components of $\left\langle v_{x}\right\rangle$ and $\left\langle v_{z}\right\rangle$ make up a circularly polarized current component. It is natural that other high frequency components are not seen in $\left\langle v_{z}\right\rangle$ because our simulation system is two-and-one-half dimensional so that the density modulation along the $z$-axis is not resolved.

In order to examine the acceleration and thermalization processes of the beam electrons, the background electrons and ions due to the interaction with the field, phase-space plots of the super-particles are shown in Figs. 6 and 7. Figures 6(a) and 6(b) show beam electrons, while Figs. 7(a) and 7(b) show background electrons and ions, respectively. In each figure, five panels with five different times, $\Omega_{\mathrm{e}} t=0,5.12$, $10.24,15.36$ and 20.48, are given. It is clearly seen in Figs. 6(a) and 6(b) that the beam electrons spread through both spatial and velocity coordinates as time elapses. They undergo slow diffusion from the center in a perpendicular direction ( $x$-axis). Note 

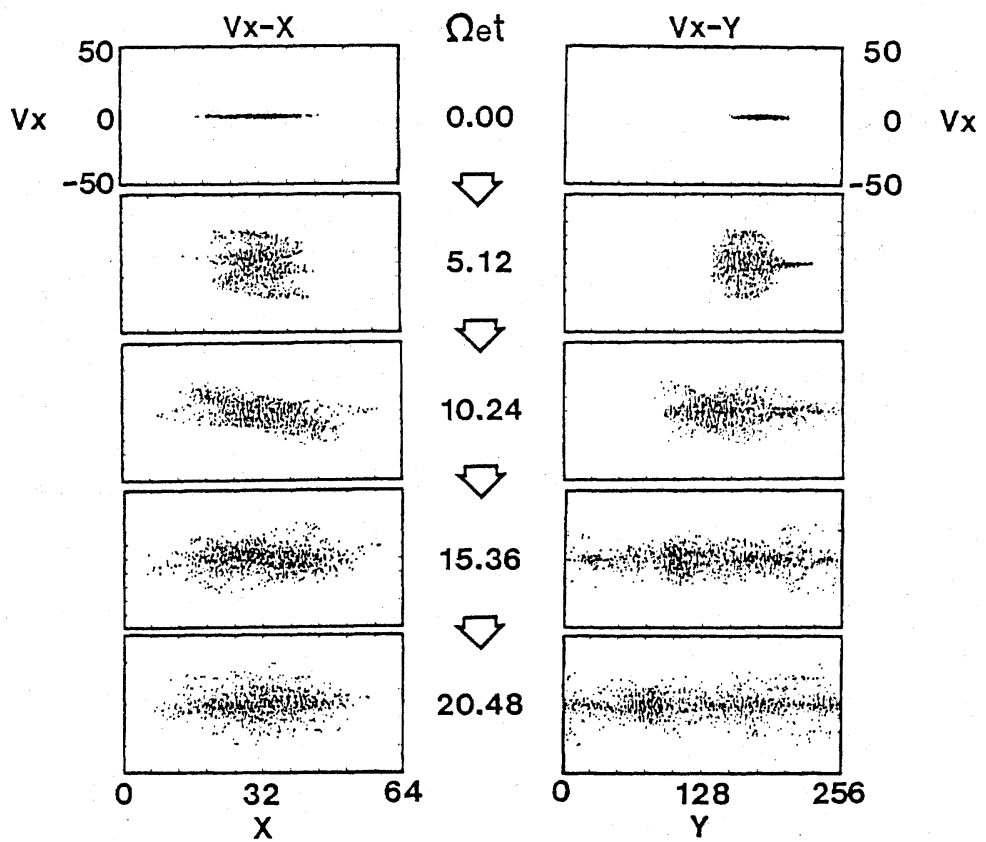

(a)
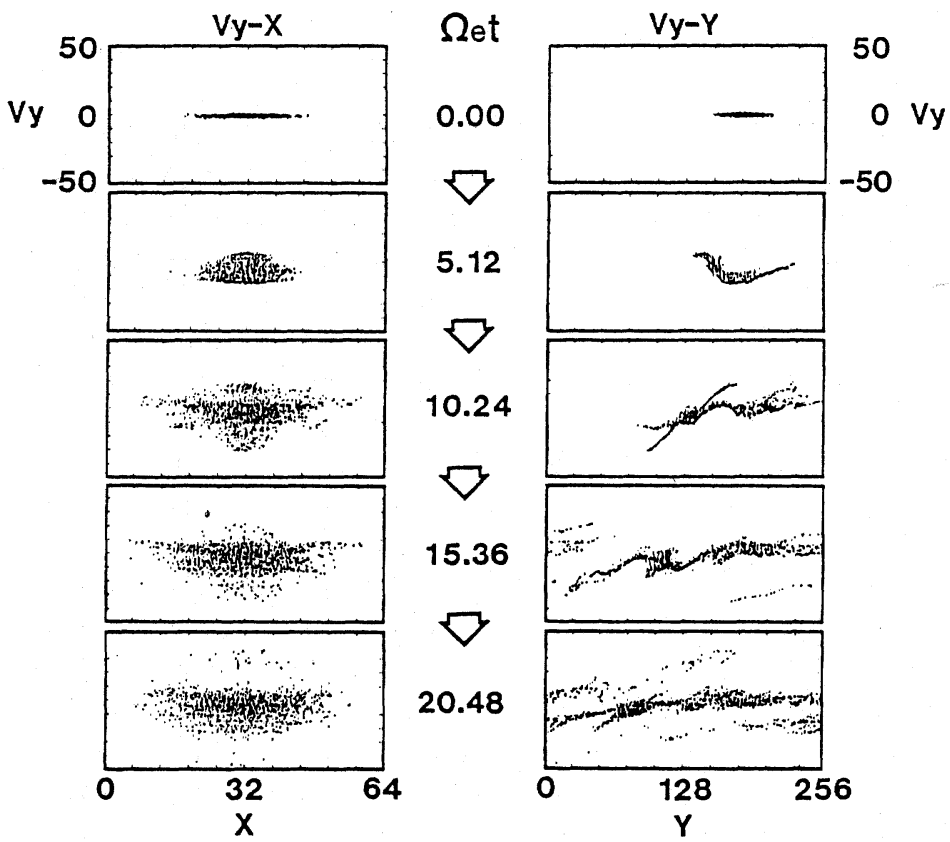

(b)

Fig. 6. Evolution of phase diagrams of the beam electrons. (a) $v_{x}-x$ and $v_{x}-y$, (b) $v_{y}-x$ and $v_{y}-y$. 

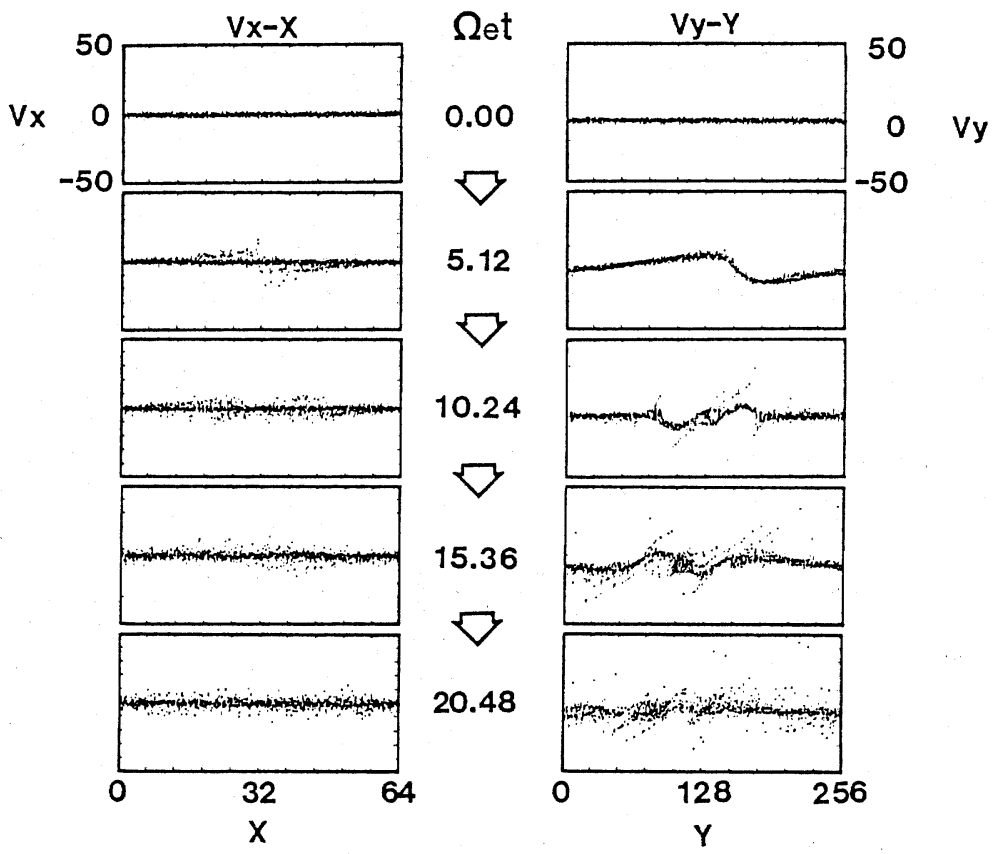

(a)
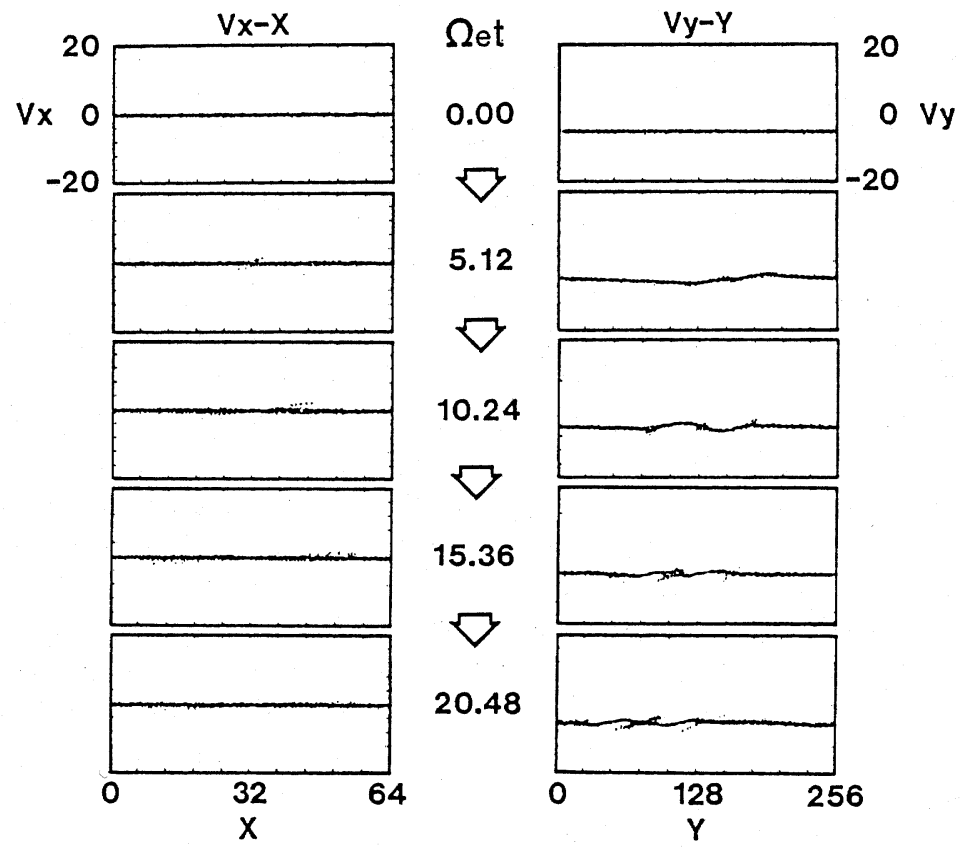

(b)

Fig. 7. Evolution of phase diagrams for background electrons and ions. (a) $v_{x}-x$ and $v_{y}-y$ for background electrons, (b) $v_{x}-x$ and $v_{y}-y$ for background ions. 

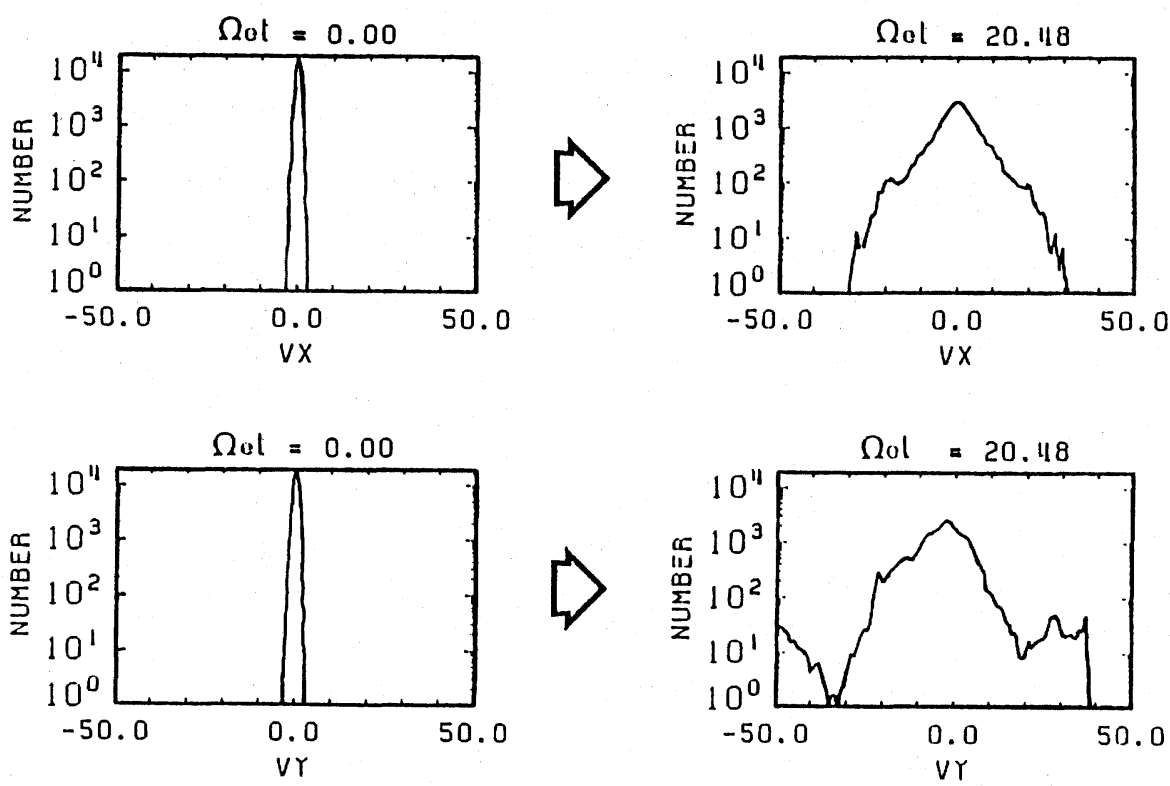

Fig. 8 .

that the width of the spread shows an oscillatory nature associated with an UHR wave. The evolution along the $y$-axis (i.e., along the external magnetic field), the deceleration of the main core of the beam and its fragmentation are clearly seen in the $v_{x}-y$ phase plot. An interesting occurrence is the appearance of a high energy tail in the beam. In addition to the deceleration of the main core of the beam and its splitting into several groups, some fraction of the beam electrons is accelerated both in the positive and negative $y$ directions. The strongest acceleration takes place at the beam center as seen in the third frame of the right column $\left(v_{x}-y\right.$ phase diagram for beam electrons). Those electrons with large positive and negative $v_{y}$ seen in the stretched branches of the third frame of the $v_{y}-y$ diagram are the remnants of the electrons accelerated at the beam center and are freely flowing in the positive and negative $y$ direction, respectively. The corresponding phase diagrams for the background electrons are shown in Fig. 7(a). The difference in the behavior of $v_{y}$ acceleration from the beam electrons is that for the beam electrons acceleration is strongest at the beam center, while that for the background electrons acceleration is strongest at the beam edges. The background ions are not accelerated much within the time range of the present simulation run; however, a small amount of acceleration in the same three places is seen as in Fig. 7(b).

The corresponding changes in the velocity distribution functions of the beam and background electrons and ions are shown in Fig. 8. The ions are not accelerated much 

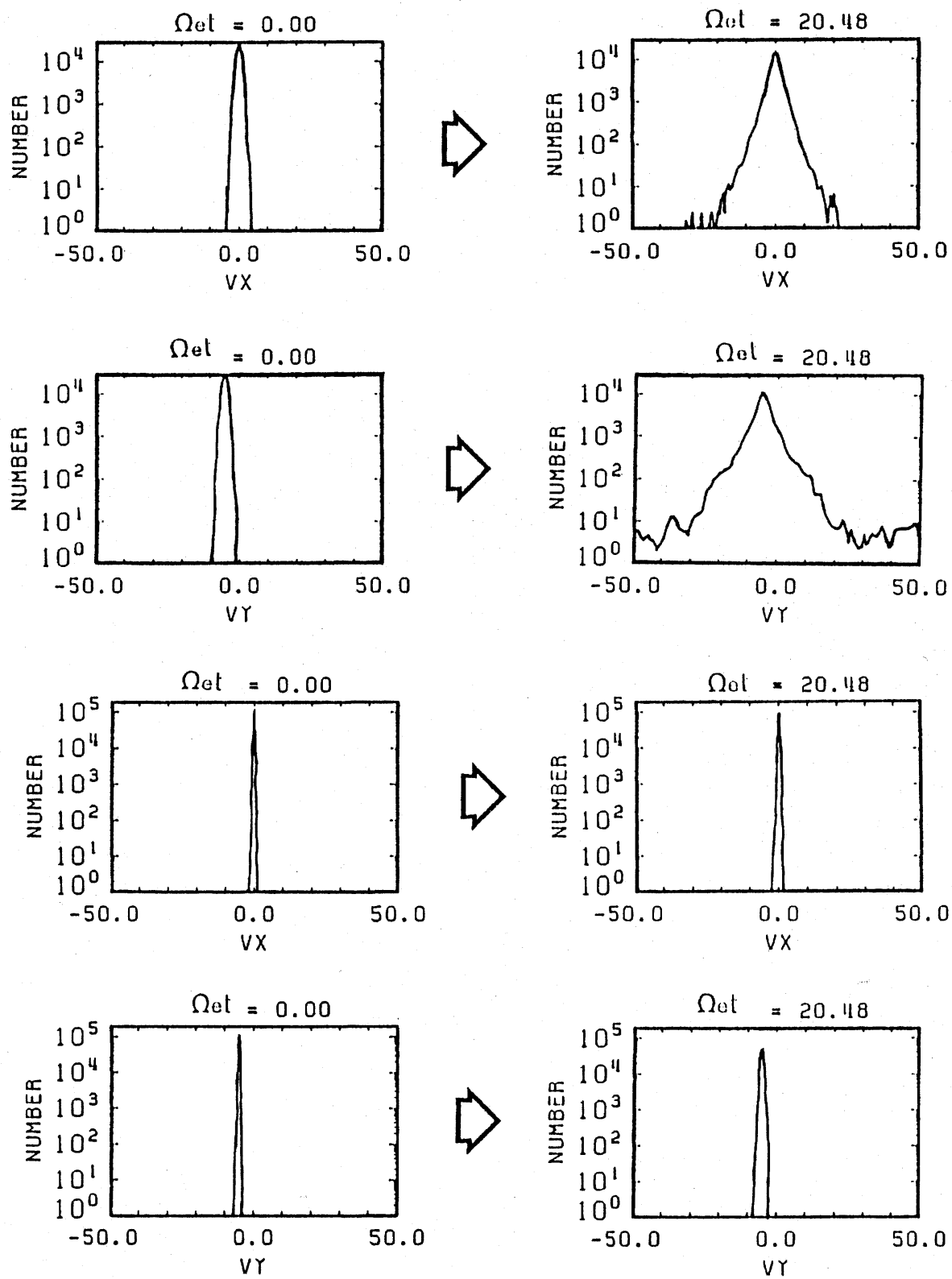

Fig. 8. Velocity distribution functions at $t=0$ and final time $t=20.48$, for beam electrons and background electrons and ions. 
as mentioned previously. It is clear that the final shapes of the distribution functions of the beam and the background electrons are different. The thermal velocity of the beam electrons in both the parallel and perpendicular directions $\left(v_{x}\right.$ and $\left.v_{y}\right)$ are much higher than the background electrons at the final time of the simulation run.

Another notable feature in the beam velocity distribution function is its asymmetry with respect to $v_{y}$ around $v_{y}=0$. Not only is the average velocity shifted toward negative $v_{y}$ but the density of the high energy electrons is also different for the different velocity signs. The beam electrons are accelerated more in the negative $v_{y}$ direction than in the positive $v_{y}$ direction. On the other hand, the density of the high energy electrons in the negative $v_{y}$ direction is lower than that in the positive $v_{y}$ direction.

Figure 9(a) shows the time evolution of the beam electron density contour map. The corresponding velocity vector plots of selected beam electrons in the same $x-y$ plane at corresponding times are shown in Fig. 9(b). Figures with the same format for background electrons are shown in Fig. 10. The electron beam which initially has an oval shape in space is elongated and finally split into many islands as is seen in Fig. 9(a). The main core of the beam is decelerated in the negative $y$ direction as discussed earlier. Acceleration perpendicular to the external magnetic field (i.e., along to the $x$-axis) takes place in an orderly fashion at $t=5.12$ but becomes random as the beam electrons are scattered at later times as is seen in the frames at $t=15.36$ and 20.48 in Fig. 9(b). For the frame where $t=15.36$ the same figure clearly shows that acceleration in the parallel direction (i.e., along the $y$-axis), takes place at the beam center, which is consistent with the $v_{y}-y$ phase space diagram shown in Fig. 7(a). The background electrons, which are uniformly distributed at $t=0$, are repelled from the area around the beam as seen in Fig. 10(a) because of the electrostatic potential centered at the beam. The density profile shows the creation of a hole in the vicinity of the main core of the beam. The subsequent change in the density profile corresponds to the change of the beam density profile. The modification of the flow velocity of the background electrons is shown in Fig. 10(b). Figure 11 shows vector plots of the electric field in the $x-y$ plane corresponding to these five times. These dynamic responses will be examined in the next section.

\section{Discussion and Conclusion}

The simulation study discussed in this paper dealt with the problem of the injection of a spatially isolated electron beam into a uniform magnetized plasma along the external magnetic field. In the simulation not only the beam electrons but also the background electrons and ions were treated as particles so that all possible kinetic effects were included. In order to treat the responses of those particles and the associated waves in direction both parallel and perpendicular to the external magnetic field, the code used was of two-and-one-half dimensions. The main features of the simulation are summarized as follows:

(1) Deceleration of the beam,

(2) Beam elongation and splitting, 
(a)
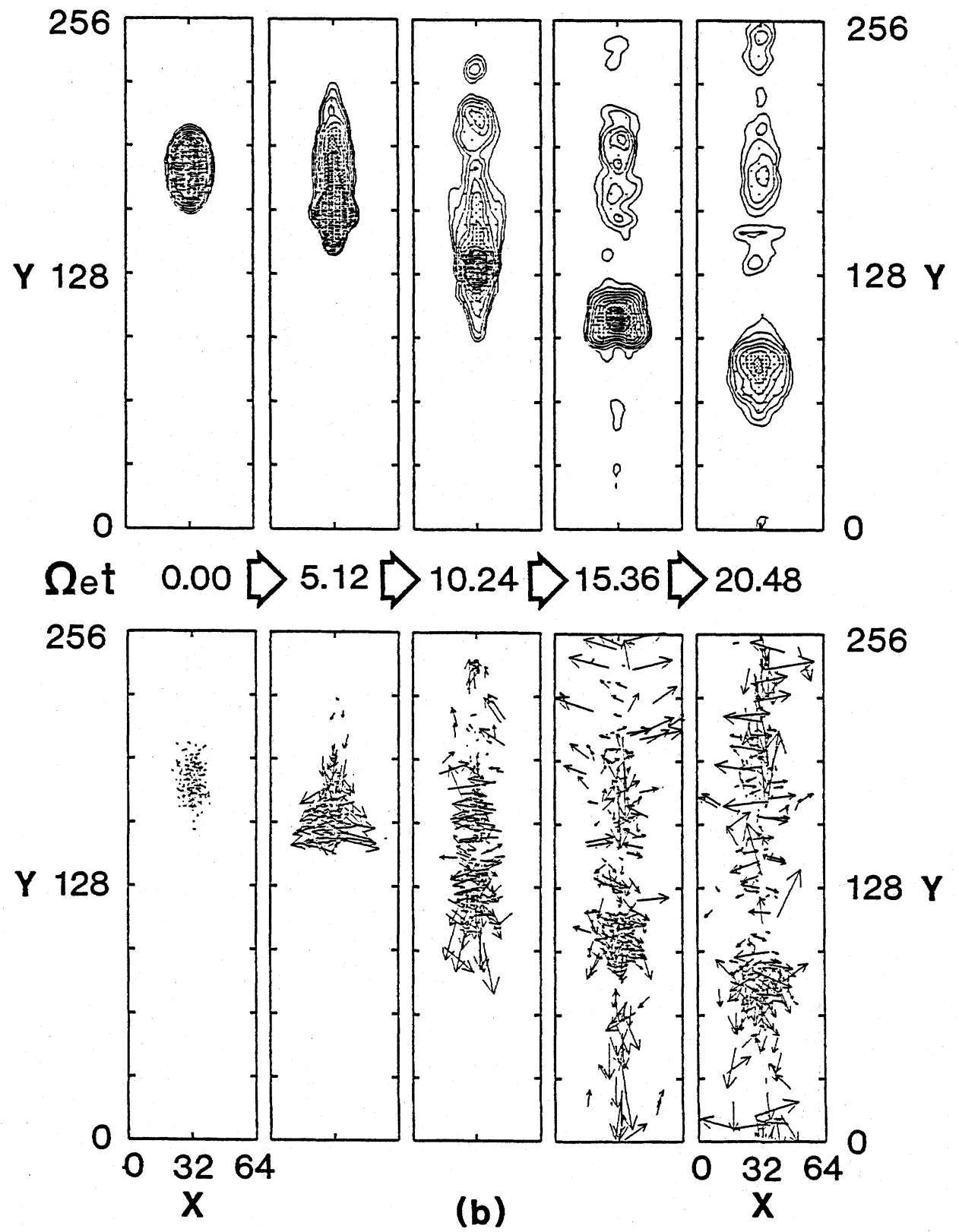

Fig. 9. Time evolution of the beam density contour map (upper panel) and corresponding velocity vector plot of selected beam electrons (lower panel). 
(a)

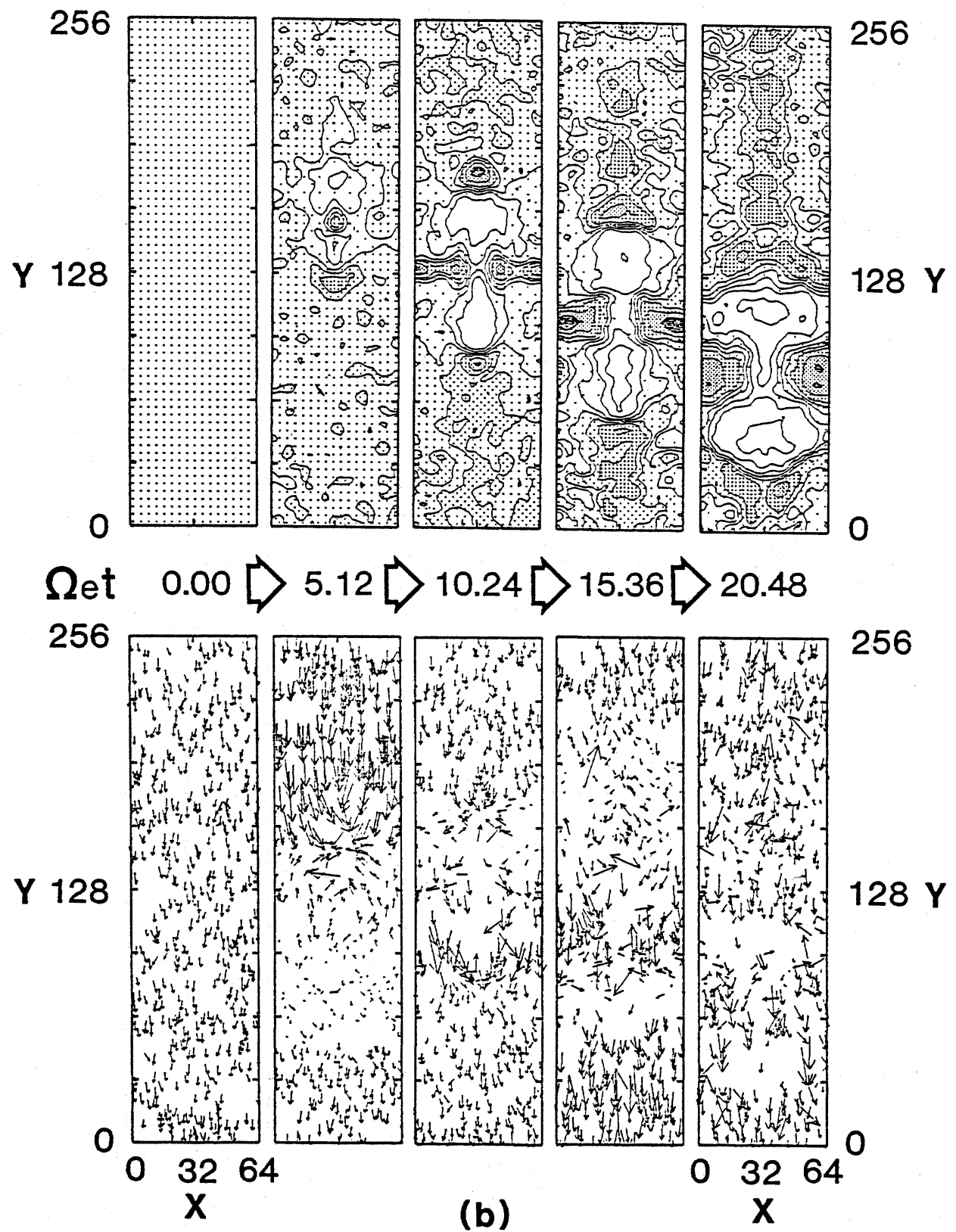

Fig. 10. Time evolution of the density contour map for background electrons (upper panel), and the corresponding velocity vector plot (lower panel). 


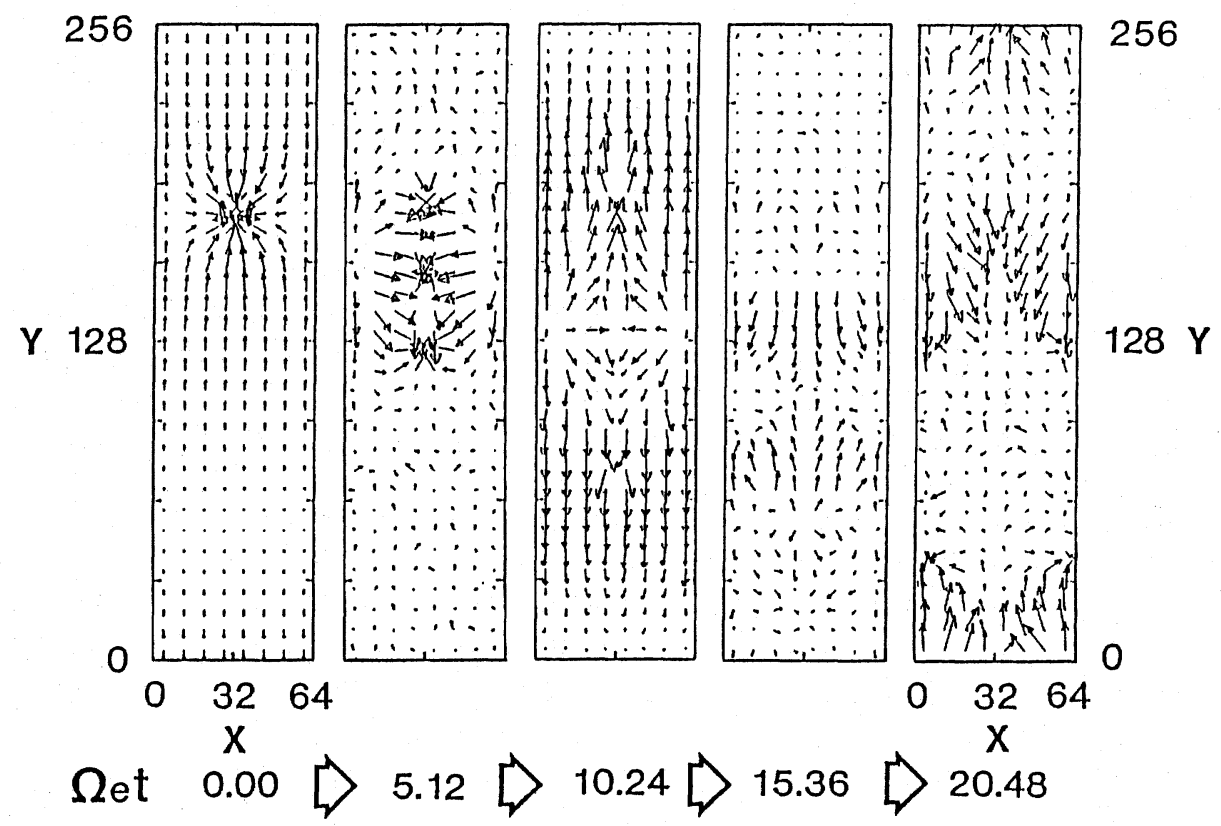

Fig. 11. Time evolution of the velocity vector plots of the electric field in the $x-y$ plane corresponding to the five times of Figs, 9 and 10.

(3) Creation of high energy tails,

(4) Thermalization,

(5) Wave emissions,

(6) Ion concentration,

(7) Creation of a quasi-steady state potential well,

(8) Creation of holes in background electrons and ions.

Many of the above features (all but \#1, \#2 and \#7) were observed in a one-andtwo-half dimensional particle simulation (MATSUMOTO and FUKUCHI, 1985). However, that simulation only treated the problem perpendicular to the external magnetic field. The present case includes the previously observed features of perpendicular acceleration, thermalization and associated UHR waves in a more complete form so as to include the competition of a perpendicular process with that in a parallel direction. Items $\# 1, \# 2$ and $\# 7$ are new results which are essentially related only to the parallel interaction. The other items also include new results related to parallel acceleration and electron plasma waves.

Regarding feature \#1 (beam deceleration), LYATSKIY (1981), assuming a steadystate and neglecting ion motion, showed that the beam speed (i.e., the beam frontal speed) should be considerably smaller or zero if the density of the beam were comparable to that of the background plasma. He argued that a potential difference 
arises at the beam front and accelerates the background electrons in the direction opposite the beam motion. The acceleration of the background electrons is accompanied by a considerable deceleration of beam particles if the beam density, and thus, the potential gap, is sufficiently large. The beam electrons reflected from the beam frontal boundary will transfer part of their energy to the drift energy of the background electrons.

Lyatskiy's steady-state view is partly consistent with our simulation results. The beam electrons, (in our case the density is sufficient to satisfy Lyatskiy's dense-beam assumption), are decelerated and finally their main core stopped. The energy transfer from the beam drift energy to the background electrons is observed in the simulation, but the transferred energy is not fed to their drift energy but to their thermal energy as seen in the distribution function in Fig. 8. Moreover, the beam electrons themselves are thermalized to a great extent, which is not interpreted nor predicted simply by the Lyatskiy model of the reflection of beam electrons from the potential well.

MALTSEV $(1982,1983)$ has shown in his one-dimensional steady-state theoretical model that the distribution function of the beam electrons trapped in the potential well, which is self-consistently produced by the injected beam, is given by

$$
\begin{aligned}
& f(v, z)=\frac{N_{0}}{\pi} \frac{\sqrt{u^{2}-v^{2}}}{V_{0}^{2}+u^{2}+v^{2}} ;-u \leqq v \leqq u, \\
& f(v, z)=0 \quad ; v \leqq-u, v \geqq u,
\end{aligned}
$$

where $u$ is the maximum velocity attainable for trapped beam electrons in the potential well and is given by $u=\sqrt{e \phi / m}$. $\phi$ is the electric potential of the well, $V_{0}$ is the speed of the potential well moving relative to the background plasma, and $N_{0}$ is the number density of the background plasma. The shape of the velocity distribution function for the case of $u \gg V_{0}$ (high beam density case) is a symmetric double peak around the drift velocity, $V_{0}$, of the well. However, his BGK type solution for the trapped electron distribution function by the Abel integral equation is based on the assumption that the background electrons are cold even in the potential well and are treated as a fluid. The ions were assumed to be an immobile background. In the present simulation, however, the distribution function of the trapped beam electrons does not show the double peak, but rather turns out to be mono-peaked and asymmetric. Moreover, untrapped beam electrons are observed to escape from the potential well supported by the main core of the beam electrons. There is also a significant heating and acceleration of background electrons. Thus, qualitatively, MAL'TSEV's theory $(1982,1983)$ is only partly consistent with our simulation. However, it can not predict other characteristics observed in this simulation such as beam elongation and splitting (item \#2), the creation of high energy tails (item \#3), and thermalization of background electrons (item \#4). This may be partly due to the differences between their theoretical model and ours.

The phenomenon of beam elongation and splitting (item \#2) is related to beam electrons which are capable of escaping from the potential well. Let us consider the 
initial response of beam and background electrons to the electrostatic electric field produced by the injection of an excess charge into a localized area. For simplicity, we will confine ourselves to a one-dimensional situation, in the direction parallel to the external magnetic field. It is noted that the initial electric potential has the form of a hill instead of that of a well. Both beam and background electrons start to expand as they slide down the potential hill. This outward motion which reduces the local negative charge, continues until a net positive charge appears in the central area of the beam. The positive charge then makes the potential turn from a hill shape to a well shape, yielding the reversed motion of those electrons in the well. Thus they exhibit oscillations with characteristic frequencies such as those of the plasma and upper hybrid frequencies in directions parallel and perpendicular to the magnetic field, respectively. From this point of view it is natural that the shape of the electron beam changes in time and results in oscillations. At the time the maximum size of the beam is reached, some of the beam electrons flow over the frontal wall of the potential well and escape. This escape takes place because the potential itself is retarded in the negative $y$ direction via a drag force due to flowing background electrons, thereby giving additional relative speed in the positive $y$ direction to the trapped beam electrons. These overflowing electrons cause elongation of the beam in the positive $y$ direction. As the main core of the trapped beam electrons is retarded in the negative $y$ direction, a gap is created between the escaped electrons and the main core of the beam resulting in beam splitting.

In the simulation it is observed that energization of both beam and background electrons takes place at three different locations along the $y$-axis. This acceleration is caused by the electrostatic electric field produced by the charge distribution along the $y$-axis. The time evolution of the $v_{y}-y$ phase space diagrams of the beam and background electrons as well as that of the $E_{y}$ electric field are shown in Fig. 12 for the period of most active acceleration. This allows a comparison of the difference in acceleration behavior between the beam and background electrons. The variations in the charge distribution that correspond to the electric field changes with time shown in Fig. 12, are shown in Fig. 13. A velocity modulation, as show in the phase diagrams, follows with a time delay of a quarter of the oscillation period of the beam and background electrons in the potential well/hill. Note that the time of the frame of the electric field is advanced by approximately a quarter of the total plasma frequency i.e., by $\Delta t=0.64(1 / 4)(2 \pi / \Pi)$ from that for the phase diagrams to show the cause-andeffect relation between them. Obviously, the background electrons are accelerated at three locations; at the beam center and at both ends of the beam. On the other hand the beam electrons are accelerated mostly at the beam center as seen in the frame at $t=9.92$. When the intensity of the electric field peaks in the vicinity of the beam center at certain phases of time (see e.g., the frame at $t=9.28$ ), both the beam and background electrons are accelerated in the positive and negative $y$ directions on the right and left sides of the beam center, respectively. However, when the electric field $E_{y}$ peaks at the edges of the beam location $(t=10.24)$ due to the appearance of sharp charge density peaks in the background electrons there, strong acceleration of the background electrons takes place in the positive $y$ direction at the left edge and in the 


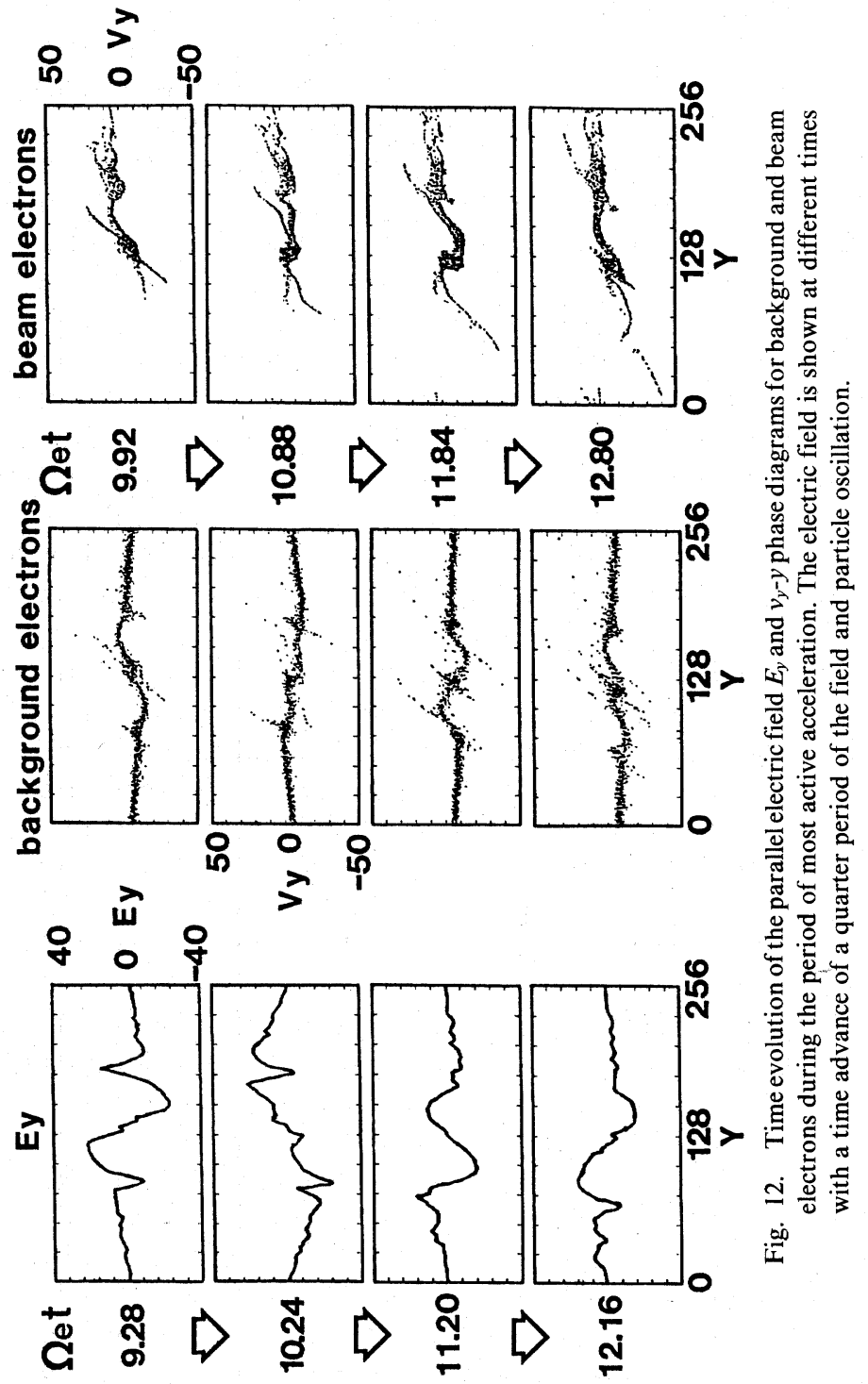




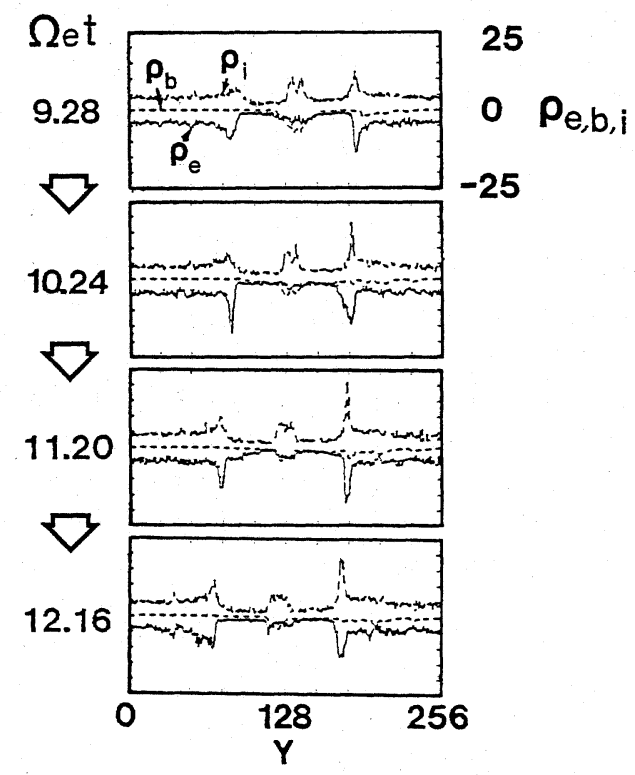

Fig. 13. Time evolution of the charge profiles along the $y$-axis at four times corresponding to those for the electric field in Fig. 12.

negative $y$ direction at the right edge of the beam (see the frame at $t=10.88$ and 11.84). The fact that very few accelerated beam electrons are found there is explained by the following two reasons; one is the low density of the beam electrons at the beam edge, and the other is that the beam electrons have already been accelerated at the beam center in the opposite direction and hence suffer only a braking effect by the electric field at the beam edges.

The thermalization process of the beam and background electrons is related to a detrapping of electrons from the potential well by acceleration. The escaping electrons flow essentially freely with different speeds along the $y$-axis. This free streaming causes a phase mixing and thus gives rise to a thermalization process.

In the theoretical treatment of spatially finite electron beams by LYATSKIY (1981) and MALTSEV $(1982,1983)$, ions are assumed to be an immobile background. However, the present simulation includes ion dynamics. In Fig. 13 the positive charge is formed by background ions, while the negative charge is attributed to background and beam electrons. The charge due to background electrons is plotted by solid line and the beam charge by a dashed line. An interesting and important feature of including ion dynamics is that the ions are attracted towards the beam center. This causes a strong acceleration of the electrons at the beam center. At a time later in the interaction, the ions are concentrated not only at the beam center but also at the beam edges, forming a charge wall at the beam edges. The BGK type steady state potential 
and charge distribution are therefore totally different from those described in the theoretical models by Lyatskiy and Mal'tsev. In our simulation, no steady state is reached but a quasi-steady state is sustained for a certain period of time. During the quasi-steady state there is little change in the charge distribution, thus maintaining quasi-neutrality. This is seen in Fig. 13. Where the background electrons and ions form one small central hump and two high-density peaks at the edges. The beam electrons form a peak at the center a plus flat distribution in the beam area, which is surrounded by two background density walls. The quasi-steady state slowly collapses at a later time. A detailed analysis of the stability of the quasi-steady state and its relation to the Lyatskiy and Mal'tsev problem will be given in a future paper.

While four different types of wave fields are generated in the simulation, here we are concerned only with the two types generating the most intense electric fields; the electron plasma waves, parallel to the external magnetic field, and the UHR waves, perpendicular to $B_{0}$. The UHR waves are the weaker of the two and contribute to beam particle acceleration and spreading in the $x$ direction. These effects are much less significant than the effects that occur as a result of plasma waves in the $y$ direction. It is noted that the acceleration and excitation of UHR waves in the perpendicular direction observed in the previous simulation (MATSUMOTO and FUKUCHI, 1985) are also seen in the present simulation. However, the dynamic reaction of the beam along the external magnetic field is more interesting as it includes the beam deformation and retardation associated with excitation of electron plasma waves. In both cases, the wave fields damp out significantly by the end of the simulation. The field energy is lost in heating and accelerating beam and background particles to a quasi-steady, quasi-neutral state and finally in restoring a peaceful charge neutrality.

The acceleration of background electrons creates a hole in the background electron distribution in the beam area. In an early phase the ions slowly move toward the center of the beam forming a peak. In the one-dimensional display of the charge density shown in Fig. 13, an electron density peak is observed in the central area of the beam at the time of the sharp ion peak formation. However, it should be stated that the central peak observed in the one-dimensional display is actually not the peak which appeared in the center of the beam area, but is a projection of the two main peaks along the $x$-axis with the $y$ coordinate located at the center of the beam. This situation is shown clearly in Fig. 10. Therefore the hole in the background electrons and ions (not shown here) is created in the center of the beam with an enhanced density wall in directions both parallel and perpendicular to the external magnetic field as can be seen in Fig. 10. The hole creation is a natural response of the plasma to restore charge neutrality, when an excess local charge is introduced by the injection of an electron beam.

We now discuss the relationship of the present simulation study and space beam experiments. As mentioned in Section 1, the present study was stimulated by the experimental facts of the SEPAC experiment (OBAYASHI et al., 1985; WILHELM et al., 1984a, b). They discovered several unexpected reactions of the ionospheric plasma when they injected their high current electron beam along the external magnetic field. 
One of them was the production of high energy electrons with very high pitch angles $\left(\alpha \sim 90^{\circ}\right)$. The energy of these secondary electrons was higher than the primary electron beam. Such an apparently unexplained fact drove our motivation of carrying out the particle simulation. The SEPAC electron beam current was high enough to inject an electron beam with a density of about 4 times higher than the ionospheric plasma density. Therefore the beam column in the vicinity of the electron gun must have carried a local excess charge. Our model of excess-charge cloud of the electron beam was thus constructed. Therefore the results of the simulation as well as the physical interpretation described above give additional information which may be useful in understanding what actually happened in the experiment. The electron beams with much less density, which have often been used in the past space experiments, may be quickly diffused both radially and longitudinally and neutralized by the background plasma. However, the electron or ion beam technique for measuring the electric field or for stimulating waves in the distant area such as in the solar wind or in the geotail will face a similar situation as treated in the present paper. We, of course, must carry out various simulational studies of similar sort by changing parameters such as the beam relative density, beam length, beam speed and beam neutrality condition (i.e., the quantity of excess charge). These studies are now under way and will be published elsewhere. The present simulation may be useful in constructing a theoretical model that includes both the transient and the steady state response of a plasma to the injection of a spatially isolated electron beam.

We thank Y. Omura for useful discussions and for helpful advice in programming. Computations were done at the Data Processing Center of Kyoto University. Part of the research was supported by an ISAS visiting scholarship (H.M.) and by the Air Force Contract F19628-85-K-0027. We would like to express our thanks to the referee for his careful reading of the paper and his constructive comments for revision.

\section{REFERENCES}

ARnoldy, R. L., C. Pollock, and J. R. Winckler, The energization of electrons and ions by electron beam injected in the ionosphere, J. Geophys. Res., 90, 5197-5210, 1985.

Baumjohann, W. and G. HAERENDEL, Magnetospheric convection observed between 0600 and 2100 LT: Solar wind and IMF dependence, J. Geophys. Res., 90, 6370-6378, 1985.

Baumjohann, W., G. Haerendel, and F. Melzner, Magnetospheric convection observed between 0600 and 2100 LT: Variation with $K_{p}, J$. Geophys. Res., 90, 393-398, 1985.

CARTWRIGHT, D. G. and P. J. KeLLOG, Observation of radiation from an electron beam artificially injected in the ionosphere, J. Geophys. Res., 79, 1439-1457, 1974.

Kawashima, N., A. Ushikoshi, Y. Murasato, A. Morioka, H. Oya, M. Ejiri, S. Miyatake, and H. MAтSUмото, Beam-plasma interaction experiment in the magnetosphere by emitting an electron beam from satellite JIKIKEN (EXOS-B), J. Geomag. Geoelectr., 33, 145-159, 1981.

Lavergnat, J., The French-Soviet experiment ARAKS: Main results, in Artificial Particle Beams in Space Plasma Studies (Proceedings of a NATO Advanced Research Institute on Artificial Beams Utilized in Space Plasma Studies held April 21-26, 1981, in Geilo, Norway), edited by B. Grandel, pp. 87-100, Plenum Press, New York, 1982.

Lyatskiy, V. B., Motion of electron beam in a cold plasma, Geomagn. Aeronomy, 21, 276-277, 1981. 
MaL'TSEv, Yu. P., Steady flow in a spatially-bounded beam of high-energy electrons in a cold plasma, Geomagn. Aeronomy, 22, 14-16, 1982.

Mal'TSEv, Yu. P., Adiabatic motion of an electron bunch in the magnetosphere, Planet. Space Sci., 31, 659-666, 1983.

Matsumoto, H. and K. Fukuchi, Computer simulation of particle acceleration and wave excitation by electron beam injection from space shuttle, Geophys. Res. Lett., 12, 61-64, 1985.

Matsumoto, H. and Y. Omura, Particle simulations of electromagnetic waves and their applications to space plasmas, in Computer Simulations of Space Plasmas, edited by H. Matsumoto and T. Sato, pp. 43-102, Terra Pub. and Reidel Co., 1985.

Matsumoto, H., S. Miyatake, and I. Kimura, Rocket experiment on spontaneously and artificially stimulated VLF plasma waves in the ionosphere, J. Geophys. Res., 80, 2829-2834, 1975.

Obayashi, T., N. Kawashima, S. Sasaki, M. Yanagisawa, K. Kuriki, M. Nagatomo, K. Ninomiya, W. T. Robbers, W. W. TAYlor, P. R. Williamson, P. BANKS, D. L. Reasoner, and J. L. Burch, Initial results of SEPAC scientific achievement, Earth-orient. Applic. Space Technol., 5, 37-45, 1985.

Omura, Y. and H. Matsumoto, Computer simulations of beam injection experiments for SEPAC/ Spacelab 1 mission, Radio Science, 20, 496-502, 1984.

Shawhan, S. D., G. B. MurPhy, P. M. Banks, P. M. Williamson, and W. J. Raitt, Wave emissions form $\mathrm{dc}$ and modulated electron beams on STS-3, Radio Science, 20, 471-486, 1984.

Wilhelm, K., W. STODEMANN, and W. RiedLER, Electron flux intensity distribution observed in responce to particle beam injection from spacelab 1, Rep. MPAE-W-70-84-09, 11 pp., Max Planck Inst., Lindau, 1984a.

Wilhelm, K., W. Stodemann, and W. Riedler, Observations of the electron spectrometer and magnetometer on board of spacelab 1 in response to electron accelerator operations, Rep. MPAEW70-84-18, 11 pp., Max Planck Inst., Lindau, 1984b.

WinCKLER, J. R., The use of artificial electron beams as probes of the distant magnetosphere, in Artificial Particle Beams in Space Plasma Studies, pp. 3-34, Plenum, New York and London, 1982.

Winckler, J. R., R. L. ARnOldy, and R. L. Hendrickson, Echo 2: A study of electron beams injected into the high latitude ionosphere from a large sounding rocket, J. Geophys. Res., 80, 2083-2095, 1975.

Winckler, J. R., J. E. Steffen, P. R. Malcolm, K. N. Erickson, Y. Abe, and R. L. Swanson, Ion resonances and ELF wave production by an electron beam injected into the ionosphere: ECHO 6, $J$. Geophys. Res., 89, 7565-7571, 1984.

Winckler, J. R., R. L. ARnOldy, and C. Pollock, The energization of electrons and ions by electron beams injected in the ionosphere, J. Geophys. Res., 90, 5197-5210, 1985. 